\title{
REPERCUSSÃO DO ISOLAMENTO SOCIAL PROVOCADO PELA PANDEMIA NAS ATIVIDADES DIÁRIAS NA DOENÇA DE PARKINSON
}

Juliana Paulino Dantas da Silva; UFPE/PPGERO - Universidade Federal de Pernambuco/ Programa de Pós Graduação em Gerontologia; juliana.paulino@ufpe.br;

Jaqueline Severo dos Santos; UFPE - Universidade Federal de Pernambuco; jaqueline.ssantos@ufpe.br;

Vinícius Barbosa de Freitas; UFPE - Universidade Federal de Pernambuco; vinicius.fsilva@ufpe.br; Izaura Muniz Azevedo; UFPE - Universidade Federal de Pernambuco; izauram73@hotmail.com; Danielle Carneiro de Meneses Sanguinetti; UFPE - Universidade Federal de Pernambuco; danielle. sanguinetti@ufpe.br;

Nadja Maria Jorge Asano; UFPE - Universidade Federal de Pernambuco; nadja.asano@ufpe.br; Maria das Graças Wanderley de Sales Coriolano; UFPE - Universidade Federal de Pernambuco; mariagracas.coriolano@ufpe.br

\section{RESUMO}

Introdução: A autonomia e independência do idoso com doença de Parkinson (DP) podem sofrer mudanças inerentes ao isolamento social. Objetivo: Avaliar a repercussão do isolamento social provocado pela pandemia da covid-19 nas atividades diárias de pessoas com DP. Métodos: Estudo transversal desenvolvido pelo Programa Pró-Parkinson, realizado por meio da ferramenta google meeting. Foram coletados dados sociodemográficos e investigada a percepção dos pacientes sobre 11 itens da seção II (atividades de vida diária) da Escala Unificada de Avaliação da DP com as seguintes possibilidades de resposta em cada item: "pior do que antes da pandemia" ou "igual a antes da pandemia". Os dados foram compilados com o software BioEstat. Resultados: Amostra composta por 20 sujeitos, com média de escolaridade correspondente ao ginásio ou ensino médio (média: $8 \pm 4$ anos de estudo), 10 homens e 10 mulheres, com média de idade $65( \pm 8)$, variando entre 52 a 76 anos, sendo 14 idosos (idade $\geq 60$ anos) e 6 adultos (idade $>50$ anos). A maioria residindo em Recife $(\mathrm{n}=8-40 \%)$ e Jaboatão $(\mathrm{n}=4-20 \%)$, aposentados $(\mathrm{n}=19-95 \%)$, vivendo com companheiro $(\mathrm{n}=13-65 \%)$. A percepção de piora durante a pandemia apresentou a seguinte distribuição nos itens: Freezing $(n=17-85 \%)$, Marcha $(n=1-75 \%)$, Vestir $(n=13-65 \%)$, Girar no leito $(n=12-60 \%)$, Cortar alimentos, higiene e tremor $(n=11$ em cada - 55\%), Escrita $(n=10$ - 50\%), Salivação $(n=9-45 \%)$, Fala $(n=8-40 \%)$ e Deglutição $(n=5-25 \%)$. Conclusão: O isolamento social provocado pela pandemia da covid-19 piorou o desempenho nas atividades diárias de pessoas com DP.

Palavras-chave: Coronavírus, Envelhecimento, Doença de Parkinson, Isolamento Social; atividades cotidianas. 